\title{
Echocardiographic Evaluation of Left Ventricular Diastolic Function after Percutaneous Coronary Intervention in Patients with Coronary Artery Disease
}

\author{
A K Mandal, A H K Chowdhury , A K Choudhury , A K M Monwarul Islam, B Guha \\ Department of Cardiology, NICVD, Dhaka.
}

Key Words:

Diastolic

function,

Percutaneous

coronary

intervention.

\begin{abstract}
Background: Although percutaneous coronary intervention (PCI) is an excellent therapy for coronary artery disease, there is a paucity of information on the efficacy of PCI in improving diastolic function, especially in Bangladesh. Because of the high prevalence of left ventricular diastolic dysfunction in coronary artery disease patients and its probable progression to heart failure, an evaluation of the role of PCI in improving diastolic function is required. Objective of the study was to evaluate the impact of percutaneous coronary intervention on left ventricular diastolic dysfunction by Doppler echocardiography in patients with coronary artery disease.
\end{abstract}

Methods: One hundred patients scheduled for elective PCI were enrolled in this study whose left ventricular systolic ejection fraction was normal or only mildly abnormal. Before PCI and 48 hours after PCI, echocardiography was done to evaluate the indices of LV diastolic function in these patients.

Results: The mean age of the patients was $52 \pm 8.6$ years, and 90 patients were male. All had mild to moderate degree of left ventricular diastolic dysfunction. Mitral $E$ wave velocity $(58.8 \mathrm{~cm} / \mathrm{s} \pm 11.8$ before treatment versus $78.1 \mathrm{~cm} / \mathrm{s} \pm 13.9,48$ hours after treatment), the peak velocity of late filling due to atrial contraction (mitral A wave velocity) $(76.6 \mathrm{~cm} / \mathrm{s} \pm 13.5$ before treatment $v \mathrm{~s} .67 .7 \mathrm{~cm} / \mathrm{s} \pm$ $15.2,48$ hours after treatment), E/A ratio (0.81 0.25 before treatment vs. $1.2 \pm 0.31,48$ hours after treatment) showed improvement after PCI. After PCI deceleration time (DT) decreased (245.6msec \pm 41.6 before treatment versus $175.5 \mathrm{msec} \pm 31.9$, 48 hours after treatment), and the difference was statistically significant $(p<0.001)$. It is notable that early diastolic mitral annular velocity (E2) improved significantly 48 hours after PCI $(5.9 \mathrm{~cm} / \mathrm{s} \pm 1.7$ before treatment vs $7.9 \mathrm{~cm} / \mathrm{s} \pm 1.6$, 48 hours after treatment, $p<0.001)$. E/ E2 ratio showed significant change 48 hours after PCI; it was statistically significant $(10.5 \pm 2.8$ before treatment vs $9.9 \pm 2.4$, 48 hours after treatment $p<0.001)$.

Conclusion: Improvement in some indices of left ventricular diastolic function after PCI indicates that PCI can be an effective treatment modality for impaired diastolic function in patients with symptomatic coronary artery disease.

(Cardiovasc. j. 2012; 4(2): 127-131)

\section{Introduction}

In the ischemic cascade left ventricular diastolic dysfunction is an important event. Left ventricular diastolic function is an important predictor of short and long term outcome in patients with coronary artery disease (CAD). Regional diastolic wall motion is impaired in ischemic myocardial segments, even when systolic contraction is preserved. Diastolic abnormalities of left ventricular (LV) relaxation and filling now appear to be the earliest manifestation of myocardial ischemia. ${ }^{1}$
Normal left ventricular systolic function at rest is evident in patients who have coronary artery disease (CAD) without previous myocardial infarction (MI). In contrast, Left ventricular diastolic filling might be impaired under resting conditions in patients with coronary artery disease but without myocardial infarction because of disturbed distensibility of the regional ischemic ventricular wall. It showed that diastolic function seemed to be more sensitive to ischemia than systolic function. ${ }^{2}$ Pulsed wave Doppler tissue imaging is a useful non-invasive technique which allows the assessment of regional

Address of Correspondence: Dr. Alok Kumar Mandal, Department of Cardiology, National Institute of Cardiovascular Diseases, Dhaka, Bangladesh. 
diastolic performance and dynamics of the left ventricular myocardium.

In last two decades there was a substantial increase in prevalence of diastolic heart failure (DHF) from $38 \%$ to $54 \% .^{3}$ Diastolic heart failure (DHF) is the major cause of morbidity and mortality all over the world. It is responsible for more than $50 \%$ of the heart failure cases. New onset of symptomatic DHF is a lethal disease with a 5-yr mortality of approximately $50 \%$. The prognosis of patients suffering from DHF is as ominous as prognosis of patients suffering from systolic heart failure (SHF). ${ }^{4}$ Therefore early recognition of DHF in relatively asymptomatic or less symptomatic patients with occult LV diastolic dysfunction is a real challenge. Early recognition of occult DHF and timely therapeutic intervention may help in prognostic stratification in DHF. ${ }^{5}$

Temporal dyssynchrony between normal and ischemic myocardium may result in impaired left ventricular relaxation and filling and regional dyssynchrony would improve after normalization of myocardial perfusion in the ischemic zone. Thus, improved global left ventricular filling after PCI might result from improved regional left ventricular function. ${ }^{6}$

Improved resting LV diastolic filling after Percutaneous Coronary Intervention (PCI) may reflect a reduction or elimination of reversible myocardial ischemia. ${ }^{1}$ Left ventricular diastolic filling improves gradually after PCI, possibly reflecting the phenomenon of post ischemic "stunned" myocardium. In the setting of recurrent ischemia after AMI, an opportunity may exist during which early PCI can lead to improvement in LV filling characteristics.

The effects of PCI on left ventricular diastolic function have not been systematically investigated in patients treated for coronary artery disease (CAD) in our population. The aim of this study was to determine the effects of improved myocardial perfusion on impaired left ventricular diastolic abnormalities, and to investigate the reversibility of these diastolic abnormalities in patients with CAD in the setting of PCI.

\section{Methodology}

The study was conducted in the Department of Cardiology, National Institute of Cardiovascular
Diseases (NICVD), Dhaka, Bangladesh from January 2011 to June 2011.It was a quasiexperimental study with pre-post test design. The general objective of the study was to evaluate the impact of percutaneous coronary intervention on left ventricular diastolic dysfunction by Doppler echocardiography in patients with coronary artery disease. The specific objectives were to compare the Doppler echo indices of LV diastolic function before PCI with those of Doppler echo indices of LV diastolic function after PCI. The Institutional Review Board approved the study protocol and informed consent was obtained from each subject. A total of 100 patients were studied. Patients of coronary artery disease with LV diastolic dysfunction undergoing elective PCI in native coronary artery lesions in NICVD were considered for the study. Exclusion criteria were congenital, valvular or hypertensive heart disease, cardiomyopathies, prior PCI, prior coronary artery bypasses graft operation, patients with graft vessel stenosis or stent re-stenosis, ECG evidence of left ventricular hypertrophy.

Detailed history was taken, and clinical examination was performed. Baseline investigations like $\mathrm{Hb}$, 12-lead ECG, chest skiagram, blood sugar, serum creatinine, serum lipid profile, and echocardiography were done. Demographic profile, including age, sex, height, weight, was noted. Risk factor profile included hypertension, diabetes, dyslipidaemia, smoking and family history of premature CAD. Clinical profile included pulse, blood pressure, angina pectoris (stable or unstable), MI (at the index admission or prior), congestive cardiac failure, cardiac arrhythmias, peripheral vascular disease, stroke and functional status (NYHA Class 1 to Class 4). Trans-thoracic echocardiographic studies were obtained at 2 hours before and 48 hours after PCI. Patients were evaluated by 2D, M-mode and Doppler echocardiography. Standard echocardiographic measurements were done and averaged in 4 cardiac cycles. Echocardiographic variables specially included left ventricular ejection fraction (LVEF), pulsed wave Doppler assessment of mitral valve inflow patterns in apical 4-chamber view (peak early (E) and peak late (A) flow velocities, E/A ratio, and deceleration time of early mitral flow velocity, DT ), Tissue Doppler Imaging (TDI) assessment at the medial mitral annulus in apical 
4-chamber view (mitral annular diastolic velocity (EA i.e. E2 ), and E/E2 ratio).

Pre-procedural angiographic variables including number of stenosed vessel (single/double/triple), and degree of stenosis (0 to 100\%) were noted. Observations during the procedure included changes in minimal luminal diameter, changes in TIMI flow grades. To assess the reproducibility of Doppler measurements 8 of the 100 recordings were randomly selected and analyzed on 2 separate occasions for intraobserver variability and blindly by a second echocardiographer for interobserver variability.

\section{Observations and Results}

Table I

Distribution of the study populations by age $(n=100)$.

\begin{tabular}{lcc}
\hline Age group (years) & \multicolumn{2}{c}{ Study subjects } \\
& Mean age $52 \pm 8.6$ years \\
\cline { 2 - 3 } & Number & $\%$ \\
\hline $30-39$ & 5 & $5 \%$ \\
$40-49$ & 27 & $27 \%$ \\
$50-59$ & 45 & $45 \%$ \\
$60-69$ & 21 & $21 \%$ \\
$70-79$ & 2 & $2 \%$ \\
\hline
\end{tabular}

Table I showed that the frequency distribution of the study patients by age. The study included 100 patients and the mean age was $52.0 \pm 8.6$ years with range from 30 to 70 years. Nearly half $45(45 \%)$ of the patients was found in the age group of 50-59 years.

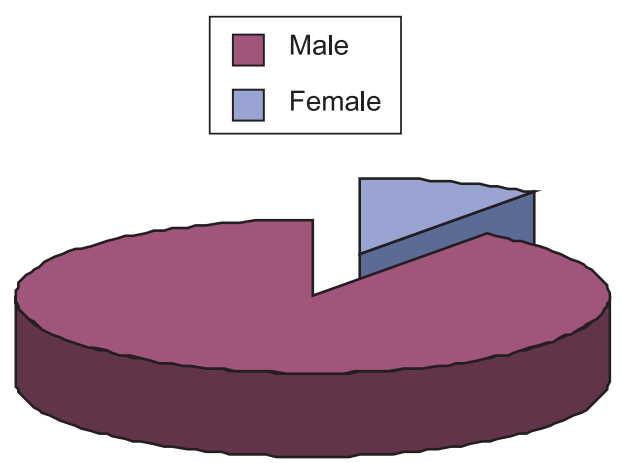

Fig.-1: Distribution of the study populations by sex $(n=100)$.
Figure 1 showed that number of male patient was $90(90 \%)$ and female patient was $10(10 \%)$. Male female ratio was $9: 1$ in the whole study population.

\section{Table II}

Risk factors distribution of the study populations $(n=100)$.

\begin{tabular}{lcc}
\hline Risk factors & \multicolumn{2}{c}{ Study subjects } \\
\cline { 2 - 3 } & Number & $\%$ \\
\hline Smoking habit & 68 & 68.0 \\
Dyslipidaemia & 50 & 50.0 \\
Family history of CAD & 46 & 46.0 \\
Hypertension & 23 & 23.0 \\
Diabetes mellitus & 19 & 19.0 \\
\hline
\end{tabular}

Table II showed that smoking was the most common risk factor (68.0\%) followed by dyslipidaemia (50.0\%), family history of CAD $(46.0 \%)$, hypertension $(23.0 \%)$, and diabetes mellitus (19.0\%) among the study patients.

\section{Table III}

Distribution of study patients by left ventricular ejection fraction $L V E F \%(n=100)$.

\begin{tabular}{lcc}
\hline LVEF\% & Number $(\mathrm{n}=100)$ & $\%$ \\
\hline $30 \%-50 \%$ & 51 & 51.0 \\
$>50 \%$ & 49 & 49.0 \\
Mean \pm SD & & $52.89 \pm 7.8$ \\
Range & & $36 \%-73 \%$ \\
\hline
\end{tabular}

Table III showed that left ventricular ejection fraction (LVEF\%) 30\%-50\% was found in 51 patients and left ventricular ejection fraction (LVEF\%) $>50 \%$ was found in 49 patients. Mean percent of ejection fraction was $52.89 \pm 7.8$ with range from $36 \%$ to $73 \%$.

Table IV

Distribution of study populations by number of coronary artery involvement $(n=100)$.

\begin{tabular}{lcc}
\hline Vessel involved & \multicolumn{2}{c}{ Study subjects } \\
\cline { 2 - 3 } & Number & $\%$ \\
\hline Single vessel disease (SVD) & 39 & 39.0 \\
Multi vessel disease & 45 & 45.0 \\
Double vessel disease (DVD) & & \\
Triple vessel disease (TVD) & 16 & 16.0 \\
\hline Total & 100 & 100.0 \\
\hline
\end{tabular}

Table IV showed that double vessel disease (DVD) was found in $45 \%$ patients followed by single vessel disease (SVD) 39\% patients, and triple vessel disease (TVD) 16\% patients. 
Table-V

Hemodynamic Findings before and after PCI $((n=100)$.

\begin{tabular}{lccc}
\hline Haemodynamic & Before PCI & After PCI & Pvalue * \\
\hline Parameters & Mean \pm SD & Mean \pm SD & \\
Heart rate & $69 \pm 9$ & $72 \pm 12$ & 0.57 \\
Systolic BP & $125 \pm 16$ & $119 \pm 14$ & 0.03 \\
Diastolic BP & $80.4 \pm 7.4$ & $79.6 \pm 6.6$ & 0.69 \\
\hline
\end{tabular}

* Paired $t$ test was done to measure the level of significance.

Table V showed that there were no significant changes before and after PCI in heart rate and diastolic blood pressure. Systolic blood pressure decreased slightly after PCI from $125 \pm 16$ to $119 \pm 14$ $(p<0.05)$.

Table VI

Comparison of echocardiographic findings before and after PCI for CAD $(n=100)$.

\begin{tabular}{lccc}
\hline \multirow{2}{*}{$\begin{array}{l}\text { Echocardiographic } \\
\text { findings }\end{array}$} & \multicolumn{2}{c}{$\begin{array}{c}\text { Coronary artery } \\
\text { disease }(\mathrm{n}=100)\end{array}$} & Pvalue * \\
\cline { 2 - 3 } & Before PCI & After PCI & \\
\hline $\mathrm{E}(\mathrm{cm} / \mathrm{sec})$ & $58.9 \pm 11.8$ & $78.1 \pm 13.9$ & 0.008 \\
$\mathrm{~A}(\mathrm{~cm} / \mathrm{sec})$ & $76.6 \pm 13.5$ & $67.7 \pm 15.2$ & 0.001 \\
$\mathrm{E} / \mathrm{A}$ ratio & $0.80 \pm 0.24$ & $1.2 \pm 0.31$ & 0.001 \\
$\mathrm{DT} \quad(\mathrm{msec})$ & $245.6 \pm 41.6$ & $175.5 \pm 31.9$ & 0.001 \\
$\mathrm{E} 2(\mathrm{~cm} / \mathrm{sec})$ & $5.9 \pm 1.7$ & $7.9 \pm 1.6$ & 0.001 \\
$\mathrm{E} / \mathrm{E} 2$ ratio & 10.52 .8 & $9.9 \pm 2.4$ & 0.001 \\
\hline
\end{tabular}

* Paired $t$ test was done to measure the level of significance.

Table VI showed that Mitral E wave velocity (58.8 $\mathrm{cm} / \mathrm{s} \pm 11.8$ before treatment versus $78.1 \mathrm{~cm} / \mathrm{s} \pm$ $13.9,48$ hours after treatment), the peak velocity of late filling due to atrial contraction (mitral A wave velocity) $(76.6 \mathrm{~cm} / \mathrm{s} \pm 13.5$ before treatment vs. $67.7 \mathrm{~cm} / \mathrm{s} \pm 15.2,48$ hours after treatment) , E/ A ratio $(0.81 \pm 0.25$ before treatment vs. $1.2 \pm 0.31$ , 48 hours after treatment) showed significant improvement after PCI ( all $\mathrm{p}<0.05$ ). After PCI deceleration time (DT) decreased $(245.6 \mathrm{msec} \pm 41.6$ before treatment versus $175.5 \mathrm{msec} \pm 31.9$, 48 hours after treatment), and the difference was statistically significant $(\mathrm{p}<0.001)$. Early diastolic mitral annular velocity (E2) improved significantly 48 hours after PCI $(5.9 \mathrm{~cm} / \mathrm{s} \pm 1.7$ before treatment vs $7.9 \mathrm{~cm} / \mathrm{s} \pm 1.6,48$ hours after treatment, $\mathrm{p}<$ 0.001). E/ E2 ratio showed significant change 48 hours after PCI; it was statistically significant (10.5 \pm 2.8 before treatment vs $9.9 \pm 2.4,48$ hours after treatment $\mathrm{p}<0.001)$.

\section{Table VII}

Comparison of diastolic dysfunction grading before and after PCI $(n=100)$.

\begin{tabular}{lcc}
\hline Echocardiographic findings & Before PCI & After PCI \\
\hline Normal LV diastolic function & 2 & 79 \\
Grade- I LV diastolic dysfunction & 45 & 1 \\
Grade- II LV diastolic dysfunction & 51 & 19 \\
Grade -III LV diastolic dysfunction & 2 & 1 \\
Grade -IV LV diastolic dysfunction & 0 & 0 \\
\hline Total & 100 & 100 \\
\hline
\end{tabular}

\section{Discussion:}

The mean age of the patients was $52.0 \pm 8.6$ years ranging from 30 to 70 years. The majority $(90 \%)$ of them were male. Similar pattern of age distribution were reported by the studies done in BSMMU in recent years. ${ }^{7}$ The study carried out by Mallick ${ }^{7}$ showed $73 \%$ patients were male and only $27 \%$ were female. Rahman ${ }^{8}$ also showed that $88 \%$ of his study patients were male and $12 \%$ were female. This study showed that smoking (68\%) and dyslipidaemia (50\%) were most frequent risk factors, followed by family history of IHD (46\%), hypertension (23\%) and diabetes mellitus (19\%). Mahajan et al. ${ }^{9}$ showed in their study that hypertension was the most common risk factor in both study groups (83\% and $70 \%)$ followed by dyslipidaemia (64\% and 68\%) and diabetes mellitus (40\% and 30\%).

The present study also showed that majority of study population had normal or mildly abnormal left ventricular ejection fraction. The mean percent of ejection fraction was $52.89 \pm 7.8$.This finding was consistent with the report of Seyed et al. ${ }^{10}$

There were no significant changes before and after PCI in heart rate and diastolic blood pressure. Systolic blood pressure decreased slightly after PCI from $125 \pm 16$ to $119 \pm 14$ ( $\mathrm{p}<$ 0.05). Since resting ejection fraction, heart rate, and blood pressure were unchanged after PCI, major changes in left ventricular end-diastolic volume did not occur or contributed importantly to increased rate of diastolic filling after PCI. Our data suggest that improved left ventricular diastolic filling after PCI is not dependent on changes in ventricular volume, but reflects either reduced myocardial ischemia or improved coronary flow. 
All the study patients exhibited abnormal diastolic filling patterns before PCI characterized by prolonged mitral deceleration times (DT), decreased E/A peak velocity ratio and increased atrial filling fraction (mitral A wave velocity). At 48 hours after PCI mitral deceleration times (DT) were significantly shortened. Concomitantly, E/A ratio increased significantly and atrial filling fraction (mitral A wave velocity) significantly decreased in all the study patients.

The early diastolic mitral annular velocity (E2 ) was significantly improved after PCI in all CAD patients and mitral E wave velocity-to - early diastolic mitral annular velocity in the medial annulus (E/E2 ratio) exhibited significant difference post PCI, in entire CAD group. The peak systolic (S) shortening velocity and the early diastolic (E2) lengthening velocities are considered to be sensitive measures of LV systolic or diastolic function. Especially, the ratio of early mitral valve flow velocity (E) divided by E2 correlates closely with LV filling pressures. Because of the absence of pseudonormalization on tissue Doppler imaging (TDI) lengthening velocity measurements, the use of blood flow Doppler measures of diastolic LV function is no longer recommended as a first-line diagnostic approach to diastolic LV dysfunction. Only when TDI lengthening velocities are suggestive but non-diagnostic or when plasma levels of natriuretic peptides are elevated, then the simultaneous presence of a low E/A ratio and a prolonged DT provide diagnostic evidence of diastolic LV dysfunction. ${ }^{5}$

\section{Limitations of the study}

The pattern of left ventricular diastolic filling is influenced by a number of factors, including left atrial pressure and compliance, left ventricular pressure and compliance, and heart rate. Thus, an obvious limitation of the present study is the lack of invasively derived variables, such as chamber stiffness, left ventricular and atrial pressures as well as pulmonary capillary wedge pressure (as an indicator of preload conditions) was not measured during the post PCI study.

The possibility of spontaneous recovery in both unstable angina and AMI patients cannot be ruled out completely, particularly in studies done relatively soon after acute chest pain syndromes.

\section{Conclusion}

From the findings of the present study it can be concluded that abnormalities of left ventricular diastolic function occur frequently in patients with coronary artery disease and Doppler echocardiography is a useful method for assessing left ventricular diastolic dysfunction in patients with coronary artery disease and recovery of diastolic function after PCI. Improvement in some indices of left ventricular diastolic function after PCI indicates that PCI can be an effective treatment modality for impaired diastolic function in patients with symptomatic coronary artery disease.

Financial Disclosure: None.

\section{References:}

1. Bonow RO et al. Improved left ventricular diastolic filling in patients with coronary artery disease after percutaneous transluminal coronary angioplasty. Circulation 1982; 66:1159-1167.

2. Inoue T et al. Left Ventricular Diastolic Filling in Patients with Coronary Artery Disease Without Myocardial Infarction. Clin. Cardiol 1991; 14:657-664.

3. Owan TE et al. Trends in prevalence and outcome of heart failure with preserved ejection fraction. $N$ Engl $J$ Med 2006; 355: 251-259.

4. Owan TE. Epidemiology of diastolic heart failure. Prog Cardiovasc Dis. 2005; 47: 320 -332.

5. Chopra HK. Diastolic Heart Failure : A Clinical Challenge Early Recognition \& Timely Intervention is the Need of the Hour. Indian Heart Journal 2009; 61(2): 138-145.

6. Green MV et al. Function of hypertrophied Left ventricular function at rest and during exercise. $A m J$ Cardiol 1983; 49 : 34-39.

7. Mallick SK. Value of ST-Segment depression with T wave inversion in lateral leads I, aVL, V4-V6 in diagnosing the left main or left main equivalent coronary artery disease. MD cardiology thesis. 2006; Bangabandhu Sheikh Mujib Medical University, Bangladesh.

8. Rahman M. Prediction of left main coronary artery obstruction by 12 lead electrocardiography in acute coronary syndromes. MD cardiology thesis. 2005; Bangabandhu Sheikh Mujib Medical University, Bangladesh.

9. Mahajan N, Hollander G, and Malik B. Isolated and significant left main coronary artery disease: demographics, hemodynamics and angiographic features. Angiology 2006; 57: 464-477.

10. Seyed RH. Evaluation of the Effect of Elective Percutaneous Coronary Intervention as a Treatment Method on the Left Ventricular Diastolic Dysfunction in Patients with Coronary Artery Disease . J Teh Univ Heart Ctr 2010; 4:194-198. 